\title{
Adolescent Systemic Hypertension: Late Diagnosis of Coarctation of the Aorta
}

\author{
Carlos Barrios and Michael Giuffre* \\ ${ }^{1}$ Department of Pediatric/Adult Echocardiographer, University of Calgary, Canada \\ ${ }^{2}$ Department of Pediatrics and Cardiac Sciences, University of Calgary, Canada \\ *Corresponding author: Michael Giuffre, Providence Pediatric Cardiology Clinic, 120, 109 Quarry Park Blvd SE, Calgary, Alberta, Canada, T2C 5E7
}

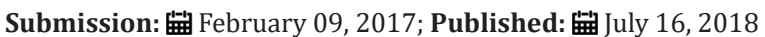

\section{Case Report}

Afourteen-year-old boy was referred to our community pediatric cardiology outpatient clinic for an incidental finding of systemic hypertension that was noted by the patient's family physician on routine evaluation. The patient was completely asymptomatic, with no visual changes, no headaches, and had a normal activity profile by history. He had an unremarkable past medical history and also had a family history of late onset hypertension.

On examination he was not dysmorphic, not in distress, was somewhat anxious, and was warm and well perfused. There was no evidence of cyanosis or clubbing. His heart rate was 80 beats per minute and regular with a respiratory rate of 20 breaths per minute. Peripheral upper limb pulses were easily palpable. Femoral and pedal pulses were decreased bilaterally, while radial pulses were notably prominent in the right and left arms. There was presence of a brachial femoral pulse delay. The blood pressures in the right and left arm were elevated at 154/70. The blood pressure in the right leg using a proper sized cuff was $80 / 58$. The chest was clear with good air entry bilaterally.

On cardiac examination, the precordium was quiet. The apex was somewhat prominent but was normally placed. There was a normal first and normally split second heart sound with no additional heart sounds. There was a harsh sounding mid pitched $2 / 6$ systolic ejection murmur heard best heard at the right and left upper sternal border, radiating toward the neck but not appreciated in the back, nor in the left or right scapular area. There was no gallop and no diastolic component to the murmur. Abdominal exam was normal with no hepatosplenomegaly.

An electrocardiogram performed at presentation showed normal sinus rhythm, normal intervals and demonstrated a frontal QRS axis of 90 degrees. The increased QRS voltages in V5 and V6 were consistent with left ventricular hypertrophy, and these voltages were accompanied by normal $\mathrm{T}$ waves.
An echocardiogram was performed. The situs was normal and there was levocardia. The subcostal images showed a blunted abdominal aortic Doppler pattern with diastolic runoff, both of which are the typical features seen in coarctation of the aorta. The parasternal short and long axis views of the echocardiogram showed normal ventricular function and moderate concentric left ventricular hypertrophy, with normal subaortic, aortic valve and supra-valve aortic anatomy. There was no intracardiac shunt defined. The suprasternal images showed colour flow acceleration in the postductal region. Careful imaging of the arch showed a normal sized ascending aorta and transverse arch, measuring $11 \mathrm{~mm}$. There was a distal discrete coarctation of the aorta measuring $5 \mathrm{~mm}$ at the isthmus. Using colour flow Doppler, turbulent flow was again noted in this region, and the continuous flow Doppler showed flow acceleration and a diastolic tail with a peak systolic gradient of $70 \mathrm{mmHg}$.

The family and patient were informed of the findings and the choices for intervention. The patient was preoperatively started on a cardio selective beta blocker and the patient was subsequently presented to a surgical center for semi-urgent coarctation repair. The surgical repair was an end to end anastomosis of the aorta and the patient had postoperative hypertension that required management with antihypertensive therapies. The patient is now followed at regular intervals to observe for resolution of the left ventricular hypertrophy and to monitor the healing and growth of the aorta, and to monitor for possible complications. Coarctation of the aorta typically presents in the neonatal or early childhood period. The severe coarctation patients are duct dependent and can become acutely unwell when the ductus closes. The less severe coarctation patients may have subtle clinical findings such as an elevated upper limb blood pressure, diminished femoral pulses, and a systolic ejection murmur that often transmits to the back. 
Coarctation of the aorta accounts for $5 \%-7 \%$ of all the cases of congenital heart disease, with an estimated incidence of approximately 3 cases per 10,000 births [1]. First described repairs of this lesion were in 1944, and since then surgical and nonsurgical techniques have developed. The morbidity and mortality rates are low for patients undergoing treatment, for both surgical or catheter interventions, though long-term follow-up is required for complications.

Today the diagnosis is often made prenatally with the advent of fetal echocardiography, however approximately $30 \%$ of neonates with coarctation remain undiagnosed upon discharge after delivery [2]. This cardiac defect generally results in left ventricular pressure overload and the patient presentation often varies with the severity of the left ventricular outflow tract obstruction. Additionally, coarctation may be associated with other cardiac defects, as well as chromosomal abnormalities such as Turner syndrome [3].

This case demonstrates that coarctation can be seen in the otherwise healthy adolescent population. The patient presented with no symptoms but had systemic upper limb hypertension and diminished femoral pulses with a brachial femoral delay, and a prominent precordial systolic murmur. The late diagnosis of adolescents and adults with coarctation of the aorta may be due to the fact that collateral circulation to the lower body developed and the first symptom may be mistaken as isolated increased blood pressure. It is crucial that a proper diagnosis occurs with appropriate investigation and management to follow. A complete clinical exam and assessment is required including a four-limb blood pressure assessment. Echocardiography and an ECG are appropriate investigations and they must be performed in a center with experience in diagnosing congenital heart disease. When the diagnosis is missed or left untreated, coarctation of the aorta can result in late aortic aneurysm formation, and/or premature coronary and cerebrovascular disease [3]. More specifically, some of the complications of coarctation of the aorta are: aortic dissection, thoracic aortic aneurysm, infective endocarditis, endocardial fibroelastosis, cerebral aneurysm rupture, impaired left ventricular systolic/diastolic function progressing to congestive cardiac failure among others [3].

It is recommended that patients with repaired and unrepaired coarctation obtain follow-up throughout their lifetime by a cardiologist, with specific attention to baseline and exercise-induced hypertension [4]. Modifications of various surgical techniques have led to low mortality and morbidity rates, and recent additions of trans catheter balloon angioplasty and endovascular stent placement have expanded treatment options [1]. Hypertension is common in post-operative patients with aortic coarctation, even in the presence of no residual coarctation, which requires appropriate follow-up and treatment.

Common Echocardiography Features of Coactation of the Aorta (Figure 1-3).
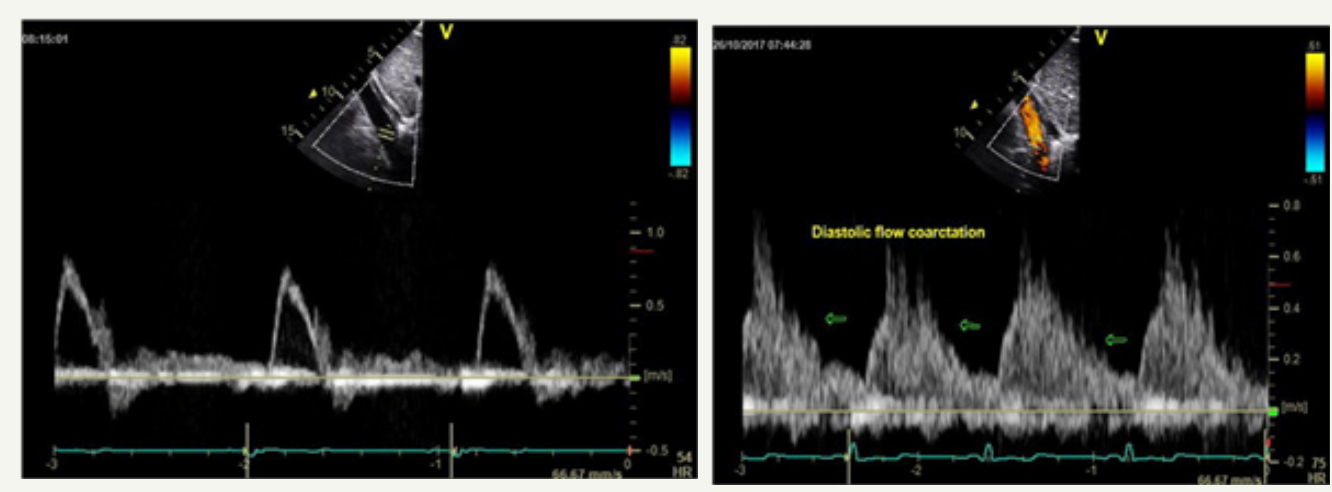

Figure 1: (Comparing the images of a normal study on the left vs the images of our fourteen-year-old patient on the right). Normal abdominal aortic flow vs Abnormal abdominal aortic flow (diastolic runoff).
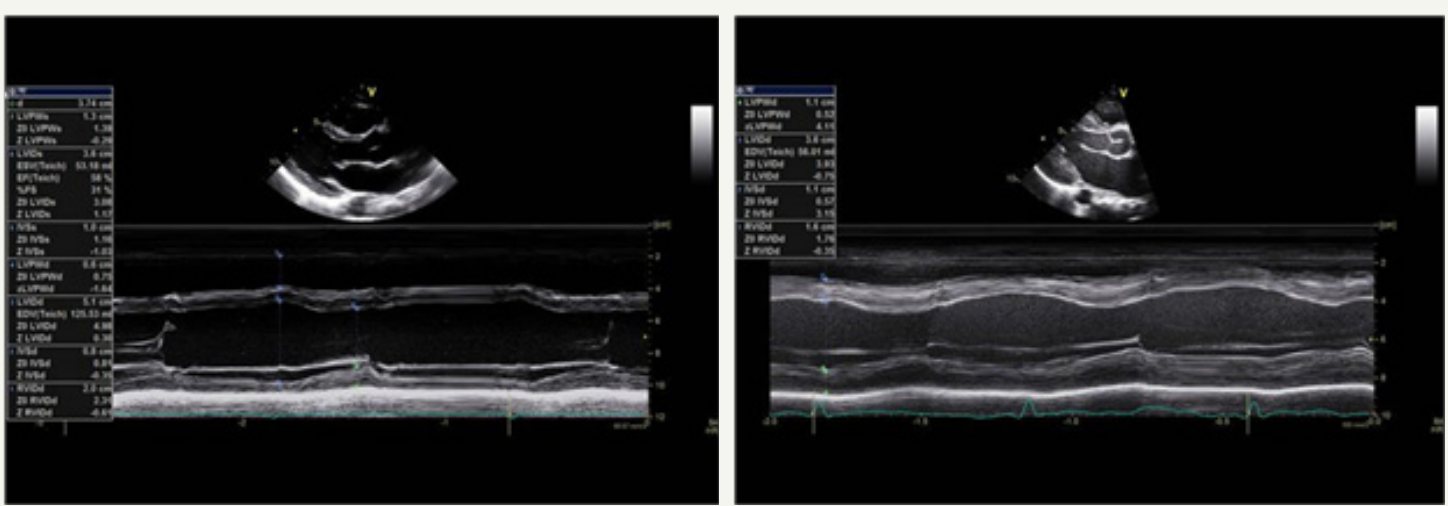

Figure 2: Normal LV wall thicknes vs. Abnormal LV wall thickness (concentric LVH). 

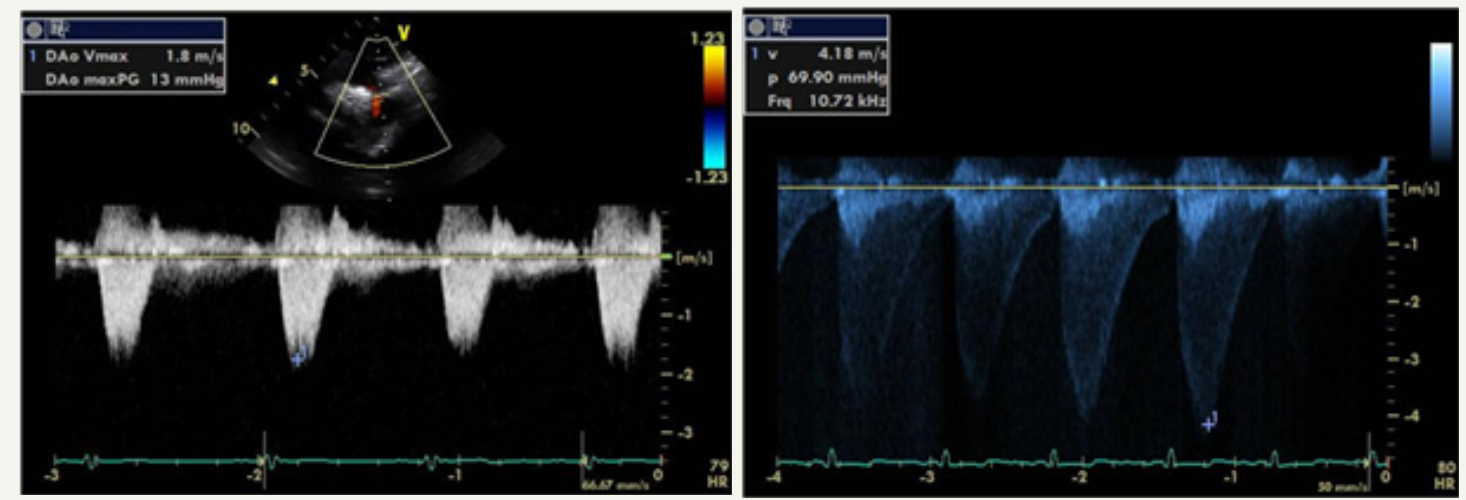

Figure 3: Normal flow pattern in descending aorta vs Abnormal flow pattern in descending aorta. Normal peak gradient $=13 \mathrm{mmHg}$

Significant increase in peak gradient $=70 \mathrm{mmHg}$.

\section{References}

1. Toroke RD, Campbell MJ, Fleming GA, Hill KD (2015) Coarctation of the Aorta: Management from infancy to adulthood. World J Cardiol 7(11): 765-775.

2. Liberman RF, Getz KD, Lin AE, Higgins CA, Sekhavat S, et al. (2014) Delayed diagnosis of critical congenital heart defects: trends and associated factors. Pediatrics 134(2): e373-e381.

3. Mcbride KL, Zender GA, Fitzgerald-Butt SM, Koehler D, MenessesDiaz A, et al. (2009) Linkage analysis of left ventricular outflow tract malformations (aortic valve stenosis, coarctation of aorta, and hypoplastic left heart syndrome). Eur J Hum Genet 17(6): 811-819.

4. Warnes CA, Williams RG, Bashore TM, John SC, Heidi MC, et al. (2008) Guidelines for the Management of Adults with Congenital Heart Disease: a report of the American College of Cardiology/American Heart Association Task Force of Practice Guidelines (writing committee to develop guidelines on the management of adults with congenital heart disease. Circulation ACC/AHA118: e714-e833.
Creative Commons Attribution 4.0 International License

For possible submissions Click Here

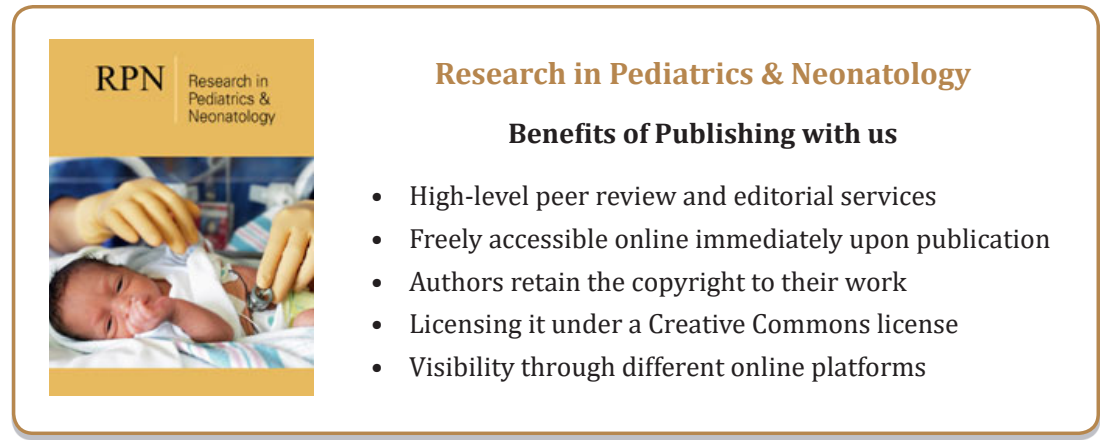

